\title{
BMJ Open What influences people's responses to public health messages for managing risks and preventing infectious diseases? A rapid systematic review of the evidence and recommendations
}

\author{
Daniela Ghio (1D , ${ }^{1,2}$ Sadie Lawes-Wickwar, ${ }^{3}$ Mei Yee Tang, ${ }^{4}$ Tracy Epton, ${ }^{2}$ \\ Neil Howlett (1) , ${ }^{5}$ Elizabeth Jenkinson, ${ }^{6}$ Sabina Stanescu, ${ }^{7}$ Juliette Westbrook, ${ }^{8}$ \\ Angelos P Kassianos, ${ }^{9}$ Daniella Watson, ${ }^{10}$ Lisa Sutherland, ${ }^{11}$ Natalia Stanulewicz, ${ }^{12}$ \\ Ella Guest, ${ }^{13}$ Daniel Scanlan, ${ }^{14}$ Natalie Carr, ${ }^{2,15}$ Angel Chater, ${ }^{16}$ Sarah Hotham, ${ }^{17}$ \\ Rachael Thorneloe, ${ }^{18}$ Christopher J. Armitage, ${ }^{2,19}$ Madelynne Arden, ${ }^{18}$ Jo Hart (1) , \\ Lucie Byrne-Davis, ${ }^{2}$ Christopher Keyworth ${ }^{2,20}$
}

To cite: Ghio D, LawesWickwar S, Tang MY, et al. What influences people's responses to public health messages for managing risks and preventing infectious diseases? A rapid systematic review of the evidence and recommendations. BMJ Open 2021;11:e048750. doi:10.1136/ bmjopen-2021-048750

- Prepublication history and additional supplemental material for this paper are available online. To view these files, please visit the journal online (http://dx.doi.org/10.1136/ bmjopen-2021-048750).

Received 07 January 2021 Accepted 05 October 2021

Check for updates

(C) Author(s) (or their employer(s)) 2021. Re-use permitted under CC BY-NC. No commercial re-use. See rights and permissions. Published by BMJ.

For numbered affiliations see end of article.

Correspondence to

Dr Tracy Epton;

tracy.epton@manchester.ac.uk

\section{ABSTRACT}

Background Individual behaviour changes, such as hand hygiene and physical distancing, are required on a population scale to reduce transmission of infectious diseases such as COVID-19. However, little is known about effective methods of communicating risk reducing information, and how populations might respond. Objective To synthesise evidence relating to what (1) characterises effective public health messages for managing risk and preventing infectious disease and (2) influences people's responses to messages.

Design A rapid systematic review was conducted. Protocol is published on Prospero CRD42020188704. Data sources Electronic databases were searched: Ovid Medline, Ovid PsycINFO and Healthevidence.org, and grey literature (PsyarXiv, OSF Preprints) up to May 2020.

Study selection All study designs that (1) evaluated public health messaging interventions targeted at adults and (2) concerned a communicable disease spread via primary route of transmission of respiratory and/or touch were included. Outcomes included preventative behaviours, perceptions/awareness and intentions. NonEnglish language papers were excluded.

Synthesis Due to high heterogeneity studies were synthesised narratively focusing on determinants of intentions in the absence of measured adherence/ preventative behaviours. Themes were developed independently by two researchers and discussed within team to reach consensus. Recommendations were translated from narrative synthesis to provide evidencebased methods in providing effective messaging. Results Sixty-eight eligible papers were identified. Characteristics of effective messaging include delivery by credible sources, community engagement, increasing awareness/knowledge, mapping to stage of epidemic/ pandemic. To influence intent effectively, public health messages need to be acceptable, increase understanding/perceptions of health threat and perceived susceptibility.
Strengths and limitations of this study

- While we conducted a rapid review, we ensured that we completed it in a systematic manner with a broad initial search (eg, no restriction on study design) to develop recommendations from lessons in risk communication that we can translate to the current pandemic.

- The rapid review included all study designs with high heterogeneity, some of which were low quality, so findings should be interpreted tentatively.

- The focus of most of the studies included in the review was on determinants of intention and not behaviour, therefore we acknowledge that the recommendations may not lead to successful enactment of target behaviours (eg, hand washing) even though they may be helpful in increasing intentions.

- We were unable to conduct backward and forward citation searching on the included studies, this may have resulted in relevant literature not being captured.

- We had strong stakeholder engagement as part of the team with different expertise of behaviour science and public health that provided feedback from initial design through to development of recommendations to be used by public health practitioners.

Discussion There are four key recommendations: (1) engage communities in development of messaging, (2) address uncertainty immediately and with transparency, (3) focus on unifying messages from sources and (4) frame messages aimed at increasing understanding, social responsibility and personal control. Embedding principles of behavioural science into public health messaging is an important step towards more effective health-risk communication during epidemics/pandemics. 


\section{BACKGROUND}

The outbreak of novel infectious diseases, including COVID-19, requires rapid changes to existing (eg, related to physical distance) and new (eg, use of face coverings) behaviours in the context of uncertainties and often rapidly evolving new knowledge. Public health messaging is one component of effective risk communication strategies to ensure sustained population level behaviour change. However, little is known about what characterises effective public health messages for dealing with infectious diseases and what factors influence the public's response.

Drawing from the behaviour change wheel (BCW ${ }^{1}$ system for intervention development, the 'COM-B' model of behaviour change proposes that public health messages should be designed as multicomponent strategies to support people's capability (the knowledge/ skills), opportunity (societal norms/physical resources) and motivation (the desire/habit) to act; factors at the heart of the BCW. ${ }^{1}$ This theory has been used in current guidance to highlight core considerations for reducing the spread of COVID-19, ${ }^{2}$ namely, increasing knowledge of personal protective behaviours (capability), signposting and making available resources (opportunity), and explaining why behaviour change is important, while mitigating emotional reactions (motivation).

When building public health campaigns or interventions, it is important to consider past evidence to build a behavioural diagnosis using COM-B; accepted modes of delivery; and an evaluation of outcomes. It is crucial to understand public health messaging in rapidly changing epidemics/pandemics and for this, a review of the evidence base is needed.

\section{Review aims}

To conduct a rapid systematic review ${ }^{3}$ and to identify and synthesise evidence in order to provide evidence-based recommendations for designing and delivering public health messages for health authorities and social care organisations dealing with infectious disease outbreaks. This review aims to identify:

1. What influences people's responses to public health messages about health-risk communication.

2. What characterises effective public health messages for managing risk and preventing disease during epidemics/pandemics.

\section{METHODS}

Rapid systematic review methods ${ }^{3}$ searches started in May 2020 and analysis was completed in July 2020. Public health messages were characterised as messages delivered by mobile technology, news broadcasts/newspapers, posters, leaflets and press conferences.

\section{Information sources}

The following databases were searched: Ovid PsycINFO, Ovid MEDLINE, PsyArXiv, OSF Preprints and healthevidence.org (search strategies are presented in online supplemental appendix 1). The protocol for this rapid systematic review is published on Prospero CRD42020188704. The SPICE criteria (Setting, Perspective, Phenomena of Interest, Comparison, Evaluation, Time Scope) was used to guide data extraction. ${ }^{4}$ Our search strategy was piloted with a scoping review to ensure that the terms were capturing all relevant literature and to also choose which databases to search. These terms were then shared within the team and with public health practitioners and behaviour science experts for feedback using an iterative process to finalise our search terms.

\section{Patient and public involvement}

This work was a rapid response to a request by Public Health Practitioners to the Health Psychology Exchange consortium. The Patient Public Involvement and Engagement group within the Health Psychology Exchange group was consulted when developing the protocol.

\section{Eligibility criteria}

All study designs were considered for inclusion (eg, systematic reviews, empirical studies) and grey literature (eg, guidelines, frameworks, and policy documents) with no date restrictions until 20 May 2020.

Papers were included if they:

1. evaluated a public health messaging intervention targeted at adults aged 18 years and above (no limitations on population or region),

2. concerned a communicable disease spread via primary route of transmission of respiratory and/or touch (human to human contact).

3. were written in English.

Papers on HIV were excluded as they involved different preventative behaviours and therefore deemed to be out of scope of the review. Papers that focused exclusively on public health messaging for vaccination uptake (intentions and uptake) during epidemic/pandemics were noted and the findings synthesised in a separate review. ${ }^{5}$

To ensure that a broad range of literature, relating to epidemics/pandemics/health crisis communication, could be captured studies were not excluded based on outcome. However, outcomes of interest included preventative behaviours (eg, hand washing, quarantining), perceptions (eg, risk), intent and awareness.

\section{Study selection}

Titles/abstracts ( $80 \%$ double screened) and full texts were screened by 15 authors (figure 1; for further breakdown of the included studies see online supplemental appendix 2 in online supplemental materials). Conflicts over inclusion (2.3\% had disagreements) were resolved through discussions with four authors (online supplemental appendix 3 provides detail on each author's roles in screening, extraction and synthesis).

\section{Data extraction}

Characteristics of the papers (eg, type of message, quality of study), the type of health risk and results were extracted (online supplemental appendix 4). Four authors (JW, 


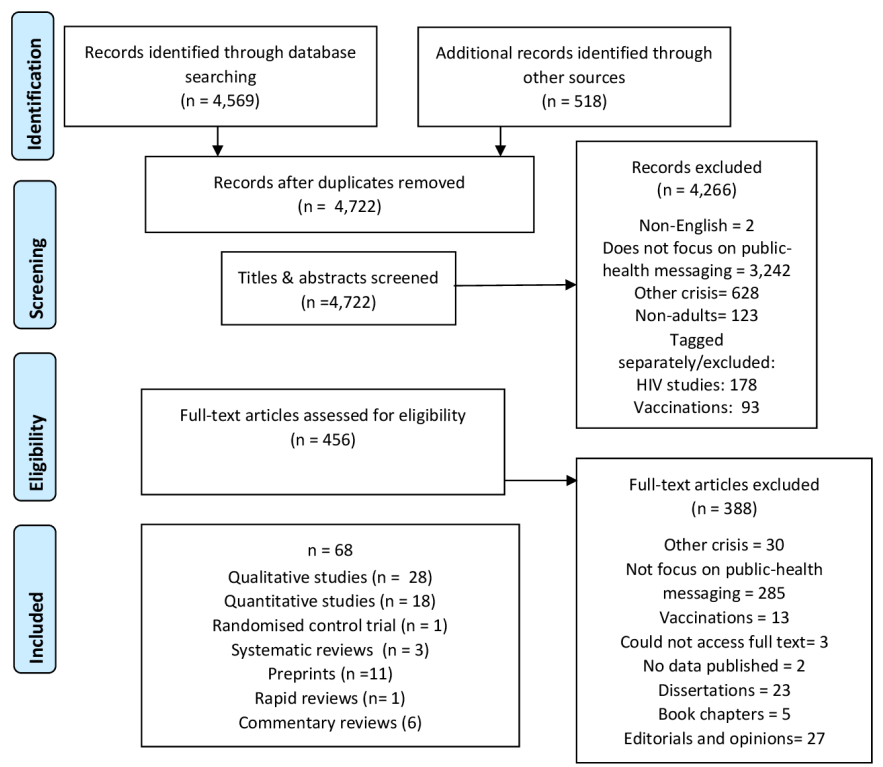

Figure 1 Preferred Reporting Items for Systematic Reviews and Meta-Analyses (PRISMA) diagram.

SS, NC and DS) screened and completed a data quality check using Mixed-Methods Appraisal Tool ${ }^{6}$ for the 54 individual papers, the 11 preprints and $\operatorname{AMSTAR}^{7}$ for the systematic reviews (online supplemental appendix 5). Overall, there was a moderate agreement level between the reviewers with $61 \%$ level of agreement. Disagreements were resolved through discussion with moderators.

\section{Synthesis of results}

A narrative synthesis was conducted ${ }^{8}$ to identify key themes with respect to: (1) what influenced people's responses to public health messages in general and for subpopulations in particular during salient time points (further details are presented in online supplemental appendix 6) and (2) interpreted recommendations for effective public health messaging for managing risk and preventing disease during epidemics/pandemics, which are presented below. The synthesis involved combining the results of reviews and individual studies reporting (1) qualitative studies, (2) quantitative studies and (3) both qualitative and quantitative studies, in order to describe the recommendations for effective delivery of public health messages. These were exported into NVivo (V.12) to data manage the combined results of different papers. To establish trustworthiness in data analysis, discussions among several members of the study team were held at fortnightly intervals to develop the coding framework, and to discuss, refine, and group the emerging codes into overall explanatory themes. All study authors were involved in establishing the conceptual framework.

\section{RESULTS}

A total of 68 papers rated as high-to-moderate quality (about $50 \%$ of them scoring as high quality and $32 \%$ of moderate quality) were included: 3 systematic reviews, 54 individual peer-reviewed papers and 11 preprints. The papers focused mainly on Influenza A virus subtype H1N1 $(n=20)$, COVID-19 $(n=15)$ and Ebola $(n=11)$ and other diseases $(n=12)$ which have emerged at different time points in the last 50 years. The timelines from initial outbreaks are highlighted in figure 2. The included studies were conducted at various time points (eg, beginning, during or post) during these pandemics and 11 did not report the timing (online supplemental appendix 6). Key variables included (1) behaviours (eg, hand washing, quarantine, using tissues, physical distancing), (2) cognitive factors (eg, increase in awareness, perceived risk) and (3) emotions (worry, anxiety) (see online supplemental appendix 5 for full characteristics of papers).

A narrative analysis of the papers was conducted on what was mostly qualitative work that reported on determinants of intent to adhere to guidelines. These were organised according to preconceptions and understanding of the threat, perceived susceptibility and perceived risk severity (threat appraisal). This narrative analysis is presented in online supplemental appendix

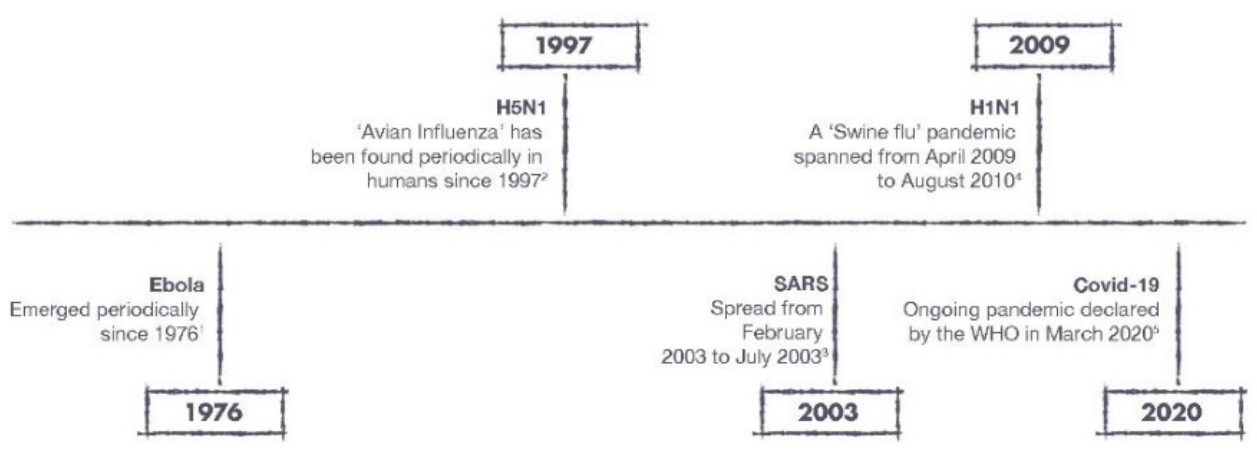

Figure 2 Timelines of pandemics/epidemics included in review. Note: 1. Centre for Disease Control and Prevention (CDC). Years of Ebola virus disease outbreak. 2019. Available from: https://www.cdc.gov/vhf/ebola/history/chronology.html; 2. WHO. H5N1 avian influenza: timeline of major events. 2012. Available from: https://www.who.int/influenza/human_animal_interface/ H5N1_avian_influenza_update.pdf; 3. CDC. CDC SARS response Timeline. 2013. Available from: https://www.cdc.gov/ about/history/sars/timeline.htm; 4. CDC. 2009 H1N1 pandemic Timeline. Available from: https://www.cdc.gov/flu/pandemicresources/2009-pandemic-timeline.html; 5. WHO. Rolling updates on COVID-19 2020. Available from: https://www.who.int/ emergencies/diseases/novel-coronavirus-2019/events-as-they-happen. 
Table 1 Recommendations mapped and cross-referenced to narrative synthesis

Recommendation

Cross-reference to narrative synthesis in online supplemental appendix 6

(1) Engaging with key stakeholders and communities

(1a) Involve community leaders and others perceived as credible sources within the community

(1b) Tailoring helps to make the key messages applicable to an individual's situation

(1c) Consider any difficulties accessing information and levels of literacy

(1d) Use different media for delivery and match delivery to the population's needs and perceptions

Community engagement

Messages for

subpopulations

Increase trust

(2) Addressing uncertainty immediately with transparency

(2a) Address uncertainty and changing information that may exist during an ongoing public health crisis

(2b) Consistency and coordination between different sources of information

(2c) Be transparent: admit errors and unknowns whenever appropriate

(2d) Be transparent: identify sources of information

(3) Unified messages

(3a) Make core messages consistent

(3b) Identify inconsistencies across sources

(3c) Increase awareness of the risks of the virus to their own health and the health of others

Increase trust

Preconceptions and understanding threat Timing - beginning of health crisis

(4) Message framing

(4a) Increase understanding of health threat

(4b) Consider social responsibility

(4c) Language choice to explain severity

(4d) Promote sense of personal control

Increase trust

Threat appraisal

Preconceptions and understanding threat

Preconceptions and understanding threat Threat appraisal Community engagement

6. Across the different themes and subthemes developed about community engagement, messages for subpopulations, increasing trust, perceptions and understanding of threat and threat appraisal, we developed four areas of recommendations to provide evidence-based steps to be taken to provide effective public health messaging during pandemics/epidemics. These recommendations are cross-referenced to the narrative synthesis in table 1 and the recommendations are reported below with evidence summarised.

\section{Recommendations}

Four recommendations were derived from the evidence (see online supplemental appendix 6 for a comprehensive report of the evidence on influences on effective public health messaging, messages for subpopulations

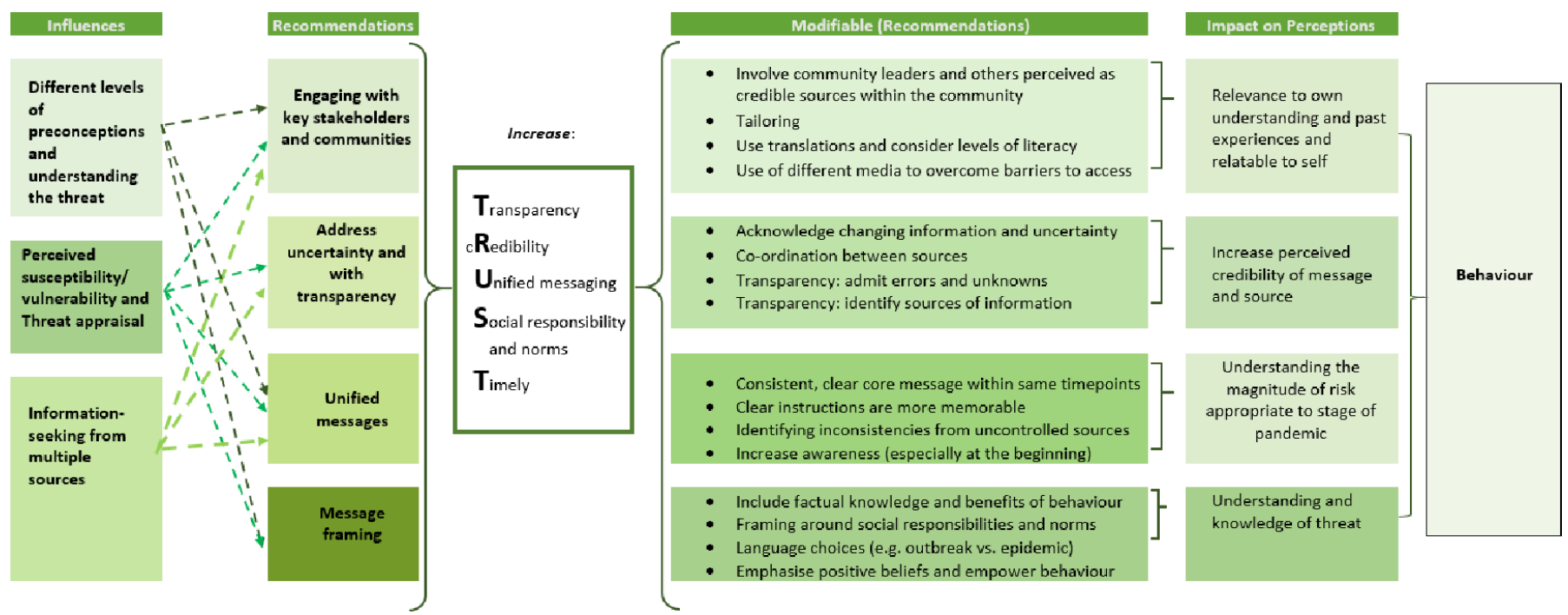

Figure 3 Diagram of synthesis of recommendations and influences on behaviour. 
and communication at salient points in an epidemic/ pandemic). Results were synthesised in figure 3, including the recommendations and influences on behaviours. The recommendations are (1) engaging with different communities, (2) addressing uncertainty immediately, with transparency, (3) unified messaging and (4) message framing.

\section{(1) Engaging with key stakeholders and communities}

(1a) Involve community leaders and others perceived as credible sources within the community

One high-quality study indicated that messages designed without input from the target population may lead to low levels of public adherence to behaviour change messages. ${ }^{9}$ A moderate-quality review found that this is especially important when dealing with inconsistencies and changing information. ${ }^{10}$

A high-quality systematic review found that the public pay more attention to messages if the community is engaged in its development ${ }^{11}$ as this will ensure that the information is relatable, ${ }^{9}$ and addresses the concerns, values, interests and priorities of the community. ${ }^{10}$ Consequently, this may lead to heightened perceptions of personal risk. Two studies of low-to-moderate quality, suggested that one way to do so is to include community leaders, to find people who are trusted ${ }^{12}$ and allow faith-based organisations to help. ${ }^{13}$ One moderate-quality study found that culturally and linguistically appropriate messages (eg, delivered via video clips) can also help retain long-term knowledge of preventative behaviours. ${ }^{14}$

A preprint study stated that over time preferred expert sources (eg, government websites) are displaced by unofficial sources (eg, social media) for information regarding epidemic/pandemics ${ }^{15}$; therefore, developing ties within the community (eg, trusted spokesperson) can be helpful to provide accurate information. A high-quality study found that students tend to perceive information from their university (from their own communities) as more credible than the media. ${ }^{16}$ One low-quality study found that community engagement is also important for quickly disseminating messages which are translated into different languages. ${ }^{1718}$

\section{(1b) Tailoring helps to make the key messages applicable to an individual's situation}

A high-quality systematic review found that those who are less likely to accept that they are at personal risk of the threat (eg, the young, least educated and hard to engage communities) are also less likely to adhere to the recommended behaviours. ${ }^{19}$ This has important implications for reducing inequalities through tailored public health messaging. Social networks and having close ties to the community are drivers of better knowledge and behaviour change. Partnership with community leaders and/or community organisations should be used to reach out to the most vulnerable (this may include those who have a disability for example, hearing/vision) and those who are least literate as well as non-Native speakers. ${ }^{19}$
Individuals desire information that fits with their experiences. ${ }^{20-23}$ Studies of moderate quality have shown that adaptable and personalised information, that is context-driven, is more effective in changing determinants of behaviour, especially in vulnerable groups. ${ }^{24} 25$ One moderate-quality study and two high-quality studies showed that messages should be tailored to take into account: (1) different levels of perceived susceptibility (eg, younger adults see themselves as less vulnerable than older adults) ${ }^{26}$; (2) likelihood of misunderstanding instructions (eg, older people thought a campaign was referring to handkerchiefs rather than disposable tissues) $^{27}$; and (3) skills needed to enact the behaviour (eg, migrant workers did not know how to wear a face covering).$^{28}$ One low-quality study found that there may be differences in message preferences (eg, older adults and mothers preferred messages that emphasised the protection of others). ${ }^{27}$

\section{(1c) Consider any difficulties accessing information and levels of literacy}

Messages are typically delivered at a high literacy level. ${ }^{19}$ When individuals do not understand the message, they may engage in behaviours that reflect their understanding. ${ }^{29}$ Some target groups, such as those with low literacy levels, could particularly benefit from a simplified, clear and consistent message. ${ }^{30}$ Pilot-testing messages can help identify phrasing that can be confusing or unfamiliar. $^{31}$

It is also important to ensure public health messages reach non-native speakers by using translated materials as shown in a high-quality study. ${ }^{32}$ Older people in nonnative groups in particular may not understand English. Thus, messages should be communicated in the original languages and through generations. Translated information should be disseminated through culturally appropriate channels such as community visits, town hall meetings and health and education and communication channels to complement mass media messages. This reinforces the need for community leaders (recommendation 1a) in aiding with translations and identifying the appropriate channels.

Some people have limited experiences of engaging in recommended behaviours (eg, using face coverings or a thermometer)..$^{33}$ These limited experiences highlight a need for training/skill development to be included as part of a public health campaign. This will improve health literacy and self-efficacy, especially when it refers to 'new' behaviours. Including training/skill development fits in with taking a COM-B model approach in developing public health messaging as it increases an individual's physical/psychological capabilities. Benefits to taking this approach could be enhanced with equally improving motivation (reflective and automatic) by considering other recommendations (eg, recommendation 4: message framing) as well as considering opportunity for behaviour (social and physical) which can identify potential barriers (eg, social norms). 
(1d) Use different media for delivery and match delivery to the population's needs and perceptions

Message delivery should be appropriate for the targeted population. For example, two studies of moderateto-high quality suggested that social media can reach younger people. ${ }^{34} 35$ A study of moderate-quality showed that social media can be effective in communicating messages fostering trust and providing opportunities for dialogue. ${ }^{36}$ However, as highlighted in two studies of high quality, there are disadvantages as (1) social media is also associated with misconceptions, such as what causes the disease ${ }^{37}$ and (2) those without internet access (or a device) would be excluded. ${ }^{38}$ A high-quality study found that those of lower socioeconomic status (SES) were less likely to use a website, and more likely to find public health messages from TV and radio to be confusing and contradictory than those of higher SES. ${ }^{30}$ In specific situations for example, messages through the use of posters in bathrooms to increase hand washing need to not just have prompts for the behaviour but also messages about transmission as a high-quality study found that prompts alone do not increase hand washing. ${ }^{39}$ This is consistent with the Health Belief Model where cues of action can trigger behaviour but requires cognitive representations of perceived susceptibility and perceived barriers/costs to action.

(2) Addressing uncertainty immediately and with transparency (2a) Address uncertainty and changing information that may exist during an ongoing public health crisis

Public health messages with emerging epidemics/ pandemics are likely to involve much uncertainty about the virus and the appropriate preventative behaviours. A high-quality systematic review found that honest reporting about the threat, through a presentation of known and unknown factors, increases people's knowledge and makes attitudes and beliefs more positive; it also increases trust in the way the government is handling the emergency. ${ }^{19}$

As epidemics/pandemics develop the information and recommended behaviours may change. Changing information decreases trust in the government. ${ }^{40}$ However, three studies of moderate-to-high quality reported that prompt (ie, giving information as it happens), stage appropriate (ie, not seen as too lenient or too extreme for the risk level) and transparent messaging (ie, that includes recommended preventative behaviours) reduces anxiety around the reported health risk. ${ }^{41}$

\section{(2b) Consistency and co-ordination between different sources of information}

Different sources of information may give information and behaviour change instructions that are inconsistent with each other, especially as knowledge rapidly changes over time. Differences in the information and behaviour change instructions between countries should be explained. Information should be transparent, not be hidden and predictions should not be too optimisticthis will increase overall trust towards authorities. ${ }^{42} 43$

\section{(2c) Be transparent: admit errors and unknowns whenever appropriate}

A high-quality study showed that if uncertainties are not acknowledged or transparency is not provided, the consequences of the errors are addressed and this can reduce trust in agencies, thus reducing information acceptance and compliance with recommendations. ${ }^{44}$

Two studies of moderate-to-high quality found that information needs to be released as early as possible at the start of the outbreak, whenever there is conflicting information and even if there are unknowns. ${ }^{45}{ }^{46}$ Honest, open $^{25}$ and explicit information, transparency and assurances of personal data safety are important ${ }^{47}$ especially when promoting preventative behaviours. ${ }^{48}$ A low-quality study found that if official sources report the outbreak before unofficial/informal sources, then they become the leading indicator that people use for their information. ${ }^{17}$ Delay can impact the public's trust in official sources that leads to beliefs that the threat is exaggerated by government or news media ${ }^{22}$ and increases the chance of apathy and communication fatigue. ${ }^{16}$

\section{(2d) Be transparent: identify sources of information}

Two high-quality studies reported that attempting to increase knowledge in the context of low levels of public trust in the source can make a message ineffective; and mistrust can increase if the perception is that the information is exaggerated ${ }^{22} 49$ or if the outbreak is perceived as uncontrolled. ${ }^{50}$ Three preprint studies reported that trust is key to the acceptance of messaging that can lead to behaviour change (eg, using masks, physical distance), thus there is greater adherence when the messages come from trusted sources. ${ }^{155152}$ Sources that potentially can be perceived as credible by the general population can include public health experts, organisations (eg, Centers for Disease Control and Prevention) and state and local governments. ${ }^{53}$ 54 $\mathrm{A}$ study found that believability of messages was reduced by scepticism towards the media and governments; in these instances, the threat was perceived as exaggerated and able to cause unnecessary panic which could influence low adoption of recommended behaviours. ${ }^{22}$ Therefore, it is important to ensure transparency highlighting that information sources are credible and legitimate. ${ }^{55}$

\section{(3) Unified messages}

(3a) Deliver consistent, clear, core messages about risk and preventative behaviour across sources within the same time points Where possible, messages should be unified across sources; as shown by two high-quality studies, this is especially important for those groups who have lower literacy (eg, lower SES) as they are more likely to perceive messages as confusing and inconsistent. ${ }^{30} 38$ A moderate-quality review found that unifying messages result in a greater understanding of the health message, greater perceived risk and 
clear guidelines for behaviour ${ }^{10}{ }^{30}$; these all contribute to increased trust. A high-quality study found that a repeated measures survey during the H1N1 pandemic, showed trust levels in the government decreased over time and this decrease was due to conflicting messages at concurrent time points. ${ }^{40}$ In two moderate-quality studies it was found that if messages from different sources provide clear, consistent instructions these are more likely to be recalled and adhered to. ${ }^{56}$ When messages provided the public with clear consistent information, while admitting that information is evolving, reported the risks, and focused on risk-reducing actions, people were more likely to perform protective behaviours (eg, clean objects, wash hands and use tissues when sneezing). ${ }^{57}$

\section{(3b) Identify inconsistencies in messages from uncontrolled} sources, especially when addressing key preventative behaviours While official sources of information (such as governmental sources, or public health bodies) are perceived as legitimate, individuals do not always find them useful as highlighted in one high-quality study and one moderatequality study, ${ }^{35}{ }^{36}$ and consequently seek other sources (eg, unofficial sources such as family or social media). In a moderate-quality study, it was found that individuals seek information from multiple sources to meet different information needs. ${ }^{45}$ Different unofficial sources of information may give information and behaviour change instructions that are inconsistent with each other and official sources. Additionally, as found in a high-quality study, unofficial sources may detract from understanding of preventative behaviours. ${ }^{58}$ It is important to acknowledge and address the inconsistencies in unofficial sources.

When addressing inconsistency, a high-quality study found that it is also important to recognise that some messages may appear inconsistent due to varying levels of personal risk of different groups (conditional messages). To improve clarity these conditional messages should be identified and where possible explained or dispelled. ${ }^{32}$

\section{(3c) Increase the public's awareness of the risks of the virus to their own health and the health of others}

Public health messaging that included information about the threat can be effective in identifying symptoms and changing behaviours. From six high-quality studies, it was found that the key aim of public health messages early in an epidemic/pandemic is typically to increase knowledge and awareness of the health-risk (see online supplemental appendix 6 for papers and priorities mapped onto time points). ${ }^{16022555960}$ However, knowledge about the virus alone is not sufficient to change behaviour ${ }^{59}$; acknowledgement of unknown factors, how to identify symptoms and how to prevent contracting and spreading the virus is also necessary as highlighted by five high-quality studies and a high-quality systematic review, two moderate-quality and one low-quality study. ${ }^{19} 22323341556162$ A low-quality study found that when perceived risk is low, ignoring recommended behaviours is rationalised. ${ }^{63}$

\section{(4) Message framing}

(4a) Increase factual knowledge of all aspects of a virus (eg, symptoms) and benefits of preventative behaviour using an appropriate message frame

Six high-quality studies found that framing and choice of language are influential in how individuals understand the threat and in turn behave. ${ }^{22}{ }^{64-68}$ Positively framed messages (emphasising the benefits of prevention behaviours) may be effective. ${ }^{64}$ For example, gain-framed signs (eg, 'stay healthy this season. Sanitise your hands') are more effective in influencing the use of hand sanitiser than signs that emphasised people's susceptibility to contamination. ${ }^{65}$

Non-narrative messages (ie, factual) are more effective than narrative messages (eg, story-telling such as a movie Contagion) in changing knowledge and perceived response efficacy related to prevention of influenza. ${ }^{68}$ Factual and scientific knowledge in messages in the media can positively influence risk perceptions. ${ }^{66}$ While formal information (presented from credible sources) increased understanding. ${ }^{67}$

\section{(4b) Consider framing messages around social responsibility and norms}

Cohesive social networks and having close ties to the community are drivers of better knowledge and compliance with preventive measures. There is some moderatequality evidence to suggest that framing messages about others' risk, in addition to your own risk, is effective in increasing information seeking. ${ }^{45}$ In a high-quality systematic review, it was found that being worried (about self or family members at risk) was an important predictor of compliance with recommended preventative behaviours, such as using tissues, hand gel and washing hands. ${ }^{19}$ The effects of worry about others at risk on compliance with preventative behaviours can potentially be amplified when combined with messages about being socially responsible. A high-quality study found framing messages with positive social responsibility to be useful for the public. ${ }^{64}$

The grey literature highlighted that developing prosocial messages and promoting positive emotional appeals, increased willingness to self-isolate especially when producing a strong, positive emotional response (such as fear). ${ }^{69}$ Prosocial framing is effective in changing behaviour; this may be due to inducing compassion, activating social norms, altruism or moral duties. ${ }^{54} 70$

\section{(4c) Choice of language needs to be clear and appropriate to} understanding the magnitude of risk

A high-quality systematic review and a moderate-quality study found that messages should sufficiently increase worry and perceived severity of risk to self and others ${ }^{19} 45$ so as highlighted in a high-quality study, recommendations are seen as proportionate ${ }^{22}$ and behaviour change is more likely to occur. ${ }^{19}$ However, in two high-quality studies, it was found that inducing too much fear has mixed results on behaviour: it can be counterproductive if this leads to panic. $^{4971}$ 
There can also be confusion about the use of technical terms. A high-quality study found that it is difficult to differentiate between pandemic influenza and seasonal influenza especially when symptoms are framed as 'flulike symptoms. ${ }^{22}$

(4d) Frame the message to emphasise positive beliefs about one's own health and that preventative behaviour is within their control

A study of moderate quality found that when messages provided the public with clear consistent information (while admitting that information and evidence is evolving), focused on the practical actions that people can take to reduce their risk and emphasised the efficacy of those actions, people were more likely to perform preventative behaviours. ${ }^{57}$ This may also further empower them and help them become socially responsible as shown in a low-quality study. ${ }^{72}$ People are more likely to follow guidelines when there are fewer perceived barriers to perform recommended behaviours, when benefits are emphasised and when contextual factors (eg, anxiety about missed work) are addressed. ${ }^{22}$

Recommendation of increasing self-efficacy through messaging is well supported in theoretical frameworks about behaviour change and risk communication for example, the Theory of Planned Behaviour, ${ }^{73}$ Health Belief Model, ${ }^{74}$ Protection Motivation Theory, ${ }^{75}$ the Health Action Process Approach, ${ }^{76}$ COM-B model ${ }^{1}$ and Social Cognitive Theory. ${ }^{77}$ Increasing self-efficacy has positive implications on planning, intention and then possibly behaviour. This review includes limited but high-quality evidence that people want messages about specific actions that they could take to protect themselves and their families during the epidemic/pandemic ${ }^{32}$ with messages that emphasise the benefits of prevention behaviours (positively framed) potentially effective in increasing uptake and compliance ${ }^{64}$ Informing the individual regarding preventative actions to stop the spread of the disease is particularly important in the early stages (see online supplemental appendix 6 for breakdown of time points). In a moderate-quality study, it was suggested that including non-health benefits may improve adherence to recommendations. ${ }^{63}$

\section{DISCUSSION}

The aim of this systematic review was to synthesis evidencebased recommendations for designing and delivering public health messaging. We found that inconsistent messages across different sources can be detrimental to any public health messaging campaigns,${ }^{79}$ which is particularly true in times of public health crisis and uncertainty. ${ }^{80}$ Miscommunication or contradictions can result in lower compliance of preventative behaviours. Literacy is fundamental to the understanding and acceptance of any public health messaging campaign. Therefore, public health messaging should focus on clarity, simplicity, transparency and unified messaging even if tailoring to different groups regardless of the level of literacy. Even when the message is understood, different populations have different barriers to address in order to change behaviour. A COM-B behavioural diagnosis ${ }^{1}$ can help to inform public health strategies (eg, lower SES groups find costs of face coverings a barrier) ${ }^{30}$ as suggested in recent British Psychological Society guidance (see online supplemental appendix 7 for how current recommendations map onto the guidance). ${ }^{2}$ Our review suggests that unified messaging can increase trust, ${ }^{45}$ build community resilience $^{32}$ and increase perceived risk and knowledge of threat. ${ }^{49}$ Furthermore, consistent messaging through different time points during an evolving pandemic are important to consider for context-specific recommendations; these were rarely considered or reported in the studies included in the review.

We found evidence of mode of delivery as an important consideration of public health messaging. Specifically, there should be careful consideration of how to communicate public health messages, which should target specific populations. For example, making use of social media platforms to target younger populations, ${ }^{16} 34$ or the radio as a delivery method for older populations. ${ }^{20}$ Translations for different cultures, and the inclusion of community leaders as part of public health message campaigns, should also be prioritised. This would ensure that all populations are reached (and not stigmatised), and the knowledge, concerns, cultural values, interests and priorities of the targeted populations are all considered. ${ }^{81}$ However, as noted in one of the studies this can be very difficult when an in-house translation service does not exist, and the rapidly evolving scientific evidence will challenge the turnaround time for developing, translating and disseminating information. ${ }^{82}$

\section{Limitations}

We acknowledge the present rapid systematic review has limitations. Although we searched multiple databases systematically it is possible that relevant research was excluded from this review since we did not have the resources to translate non-English language papers in such a short space of time or conduct backward and forward citation searching. The inclusion of unpublished literature in the review means some findings may change once these papers have been published in peer-reviewed journals. However, our inclusive approach means a range of types of messages and a variety of factors related to what influences public perceptions of messages have been identified and used to inform recommendations for messaging during current and future epidemic/ pandemics.

The aim of this rapid review was to synthesise lessons learnt from previous epidemics/pandemics to provide evidence-based recommendations about what characteristics create effective messaging. The focus of most studies was on determinants of intent and not behaviour, which may have implications on successful enactment of target behaviours. As highlighted in theories (such as health action process approach ${ }^{76}$ ), intention formation is 
part of the process and key to planning and more work is needed to understand the translation into action. Inclusion of different infectious disease (although the messaging would be of similar behaviours) may have included different contextual influences that we could not account for when synthesising the data (eg, different countries and different social norms or political influences). Furthermore, additional work is needed to understand the moderating effects of individual differences on message acceptance.

Much of our evidence is consistent with components of relevant health behaviour models (eg, Protection Motivation Theory, ${ }^{75}$ Health Action Process Approach ${ }^{76}$ and Health Belief $\mathrm{Model}^{74}$ ), with some studies reporting the use of the models in their design. These models state that people perform protective behaviours when they perceive (1) the health threat as sufficiently threatening (ie, they are vulnerable to a severe risk), (2) the recommended behaviours to be effective and (3) they have self-efficacy to perform the behaviour. We found evidence that such cognitive appraisals were important considerations for developing public health messages, but further research is needed to examine effects on behaviour change.

\section{CONCLUSIONS}

Increasing knowledge and awareness of health risks alone is unlikely to be sufficient to increase understanding of risk and subsequent risk reduction behaviours, and adherence to recommendations. Rather, taking a multifaceted approach to public health messaging which considers all relevant drivers of behaviour (social, psychological and environmental factors), is recommended. Our four key recommendations should be considered when designing and delivering public health messages: engage communities in the development of public health messaging, using credible and legitimate sources, address uncertainty immediately and with transparency, focus on unified messages from all sources and develop messages aimed at increasing understanding, inducing social responsibility and empowering personal control. These are being translated into practical guidelines for agencies. Embedding these principles of behavioural science into public health messaging is an important step towards more effective health-risk communication.

\footnotetext{
Author affiliations

${ }^{1}$ Department of Psychology, Faculty of Health and Society, University of Salford, Manchester, UK

${ }^{2}$ Manchester Centre for Health Psychology, University of Manchester, Manchester, UK

${ }^{3}$ Department of Primary Care and Population Health, University College London, London, UK

${ }^{4}$ Behavioural Science Policy Research Unit, Population Health Sciences, Newcastle University, Newcastle upon Tyne, UK

${ }^{5}$ Department of Psychology, Sports, and Geography, School of Life and Medical Sciences, University of Hertfordshire, Hertfordshire, UK

${ }^{6}$ Department of Health and Social Sciences, University of West England, Bristol, UK

${ }^{7}$ School of Psychology, University of Southampton, Southampton, UK

${ }^{8}$ Department of Psychology, University of Bath, Bath, UK
}

${ }^{9}$ Department of Applied Health Research, University College London, London, UK ${ }^{10}$ Human Development and Health, Faculty of Medicine, University of Southampton, Southampton, UK

${ }^{11}$ Behavioural Insight for Public Affairs, Ipsos Mori, Edinburgh \& London, UK

${ }^{12}$ Faculty of Health and Life Sciences, School of Applied Social Sciences, De Montfort University, Leicester, UK

${ }^{13}$ Centre for Appearance Research,Faculty of Health and Applied Sciences, University of the West of England, Bristol, UK

${ }^{14}$ Department of Communication, Policy, and Research, Education Support, London, UK

${ }^{15}$ Faculty of Health, Psychology, and Social Care, Manchester Metropolitan University, Manchester, UK

${ }^{16}$ Centre for Health, Wellbeing and Behaviour Change, University of Bedfordshire, Bedfordshire, UK

${ }^{17}$ Centre for Health Services Studies, University of Kent, Canterbury, UK

${ }^{18}$ Centre for Behavioural Science and Applied Psychology, Sheffield Hallam University, Sheffield, UK

${ }^{19}$ Manchester University NHS Foundation Trust, Manchester Academic Health Science Centre, Manchester; and NIHR Greater Manchester Patient Safety Translational Research Centre, University of Manchester, Manchester, UK

${ }^{20}$ School of Psychology, University of Leeds, Leeds, UK

Twitter Angelos P Kassianos @angkassianos and Lucie Byrne-Davis @luciebd

Acknowledgements Thank you to the wider volunteer group of the Health Psychology Exchange (HPX) a consortium in Health Psychology supporting the UK efforts during the COVID-19 pandemic. As part of the HPX there were two groups that provided feedback on the protocol, design of the recommendations and dissemination: Patient and Public Involvement group and the Public Health Practitioners group. Thank you to the British Psychological Society (BPS) Taskforce for Behavioural Science and Disease Prevention for their dissemination of the findings into a guidance document for policymakers, professionals and the public.

Contributors DG, TE, CK, MYT, SL-W designed and resolved conflicts and wrote the first draft. Conceptualisation of the study was from DG, CK, TE, MYT, SL-W, AC, SH, RT, CA, MA, JH, LB-D. Screening of titles and abstracts (1st and 2nd) was done by NH, EJ, SS, JW, APK, DW, LS, NS, EG, DS, NC, DG, MYT, SL-W and CK. Development of guidance document was done by DG, MYT, SLW, CK, TE, AC, SH, LB-D. AG and SL-W were involved in pilot data extraction. Data extraction was done by NH, EJ, SS, JW, APK, DW, LS, NS, EG, DS, NC, DG, MYT, SL-W and CK. Quality check was done by JW, SS, EG, NC, DS, DG and MYT. Analysis was done by DG, MYT, SL-W and CK. Iterations and discussions were done by DG, MYT, SL-W, CK, TE, NH, EJ, AC, SH, RT, CA, MA, JH and LB-D. DG, MYT, SL-W, CK, TE, NH, EJ, AC, SH, RT, CA, MA, JH and LB-D agreed on final categories. Writing first draft was done by DG, TE SL-W, MYT and CK.DG is the gaurantor for this study.

Funding The authors have not declared a specific grant for this research from any funding agency in the public, commercial or not-for-profit sectors.

Competing interests None declared.

Patient consent for publication Not applicable.

Provenance and peer review Not commissioned; externally peer reviewed.

Data availability statement Data are available upon reasonable request. All data relevant to the study are included in the article or uploaded as supplementary information.

Supplemental material This content has been supplied by the author(s). It has not been vetted by BMJ Publishing Group Limited (BMJ) and may not have been peer-reviewed. Any opinions or recommendations discussed are solely those of the author(s) and are not endorsed by BMJ. BMJ disclaims all liability and responsibility arising from any reliance placed on the content. Where the content includes any translated material, BMJ does not warrant the accuracy and reliability of the translations (including but not limited to local regulations, clinical guidelines, terminology, drug names and drug dosages), and is not responsible for any error and/or omissions arising from translation and adaptation or otherwise.

Open access This is an open access article distributed in accordance with the Creative Commons Attribution Non Commercial (CC BY-NC 4.0) license, which permits others to distribute, remix, adapt, build upon this work non-commercially, and license their derivative works on different terms, provided the original work is properly cited, appropriate credit is given, any changes made indicated, and the use is non-commercial. See: http://creativecommons.org/licenses/by-nc/4.0/. 


\section{ORCID iDs}

Daniela Ghio http://orcid.org/0000-0002-0580-0205

Neil Howlett http://orcid.org/0000-0002-6502-9969

Jo Hart http://orcid.org/0000-0001-9985-5137

\section{REFERENCES}

1 Michie S, van Stralen MM, West R. The behaviour change wheel: a new method for characterising and designing behaviour change interventions. Implement Sci 2011;6:42.

2 Chater AA, Byre-Davis L, Armitage CJ. Behavioural science and disease prevention: psychological guidance. British Psychological Society, 2020. Available: https://www.bps.org.uk/sites/www.bps. org.uk/files/Policy/Policy\%20-\%20Files/Behavioural\%20science\% 20 and $\% 20$ disease \%20prevention\%20-\%20Psychological\% 20 guidance $\% 20$ for $\% 20$ optimising $\% 20$ policies $\% 20$ and $\%$ 20communication.pdf

3 Dobbins M. Rapid review guidebook. Natl Collab Cent Method Tools 2017;13:25

4 Booth A. Clear and present questions: formulating questions for evidence based practice. Library Hi Tech 2006;24:355-68.

5 Lawes-Wickwar S, Ghio D, Tang MY, Tang Daniela;, Yee M, et al. A rapid systematic review of public responses to health messages encouraging vaccination against infectious diseases in a pandemic or epidemic. Vaccines 2021;9:72

6 Hong QN, Gonzalez-Reyes A, Pluye P. Improving the usefulness of a tool for appraising the quality of qualitative, quantitative and mixed methods studies, the mixed methods appraisal tool (MMAT). J Eval Clin Pract 2018;24:459-67.

7 Shea BJ, Grimshaw JM, Wells GA, et al. Development of AMSTAR: a measurement tool to assess the methodological quality of systematic reviews. BMC Med Res Methodol 2007;7:10.

8 Popay J, Roberts H, Sowden A. Guidance on the conduct of narrative synthesis in systematic reviews. A Product from the ESRC Methods Programme Version 2006;1:b92.

9 Crosier A, McVey D, French J. 'By failing to prepare you are preparing to fail': lessons from the $2009 \mathrm{H} 1 \mathrm{~N} 1$ 'swine flu' pandemic. Eur J Public Health 2015;25:135-9.

10 Barrelet C, Bourrier M, Burton-Jeangros C, et al. Unresolved issues in risk communication research: the case of the $\mathrm{H} 1 \mathrm{~N} 1$ pandemic (2009-2011). Influenza Other Respi Viruses 2013;7:114-9.

11 Schiavo R, May Leung M, Brown M. Communicating risk and promoting disease mitigation measures in epidemics and emerging disease settings. Pathog Glob Health 2014;108:76-94.

12 Lyu S-Y, Chen R-Y, Wang S-fanS, et al. Perception of spokespersons performance and characteristics in crisis communication: experience of the 2003 severe acute respiratory syndrome outbreak in Taiwan. $J$ Formos Med Assoc 2013;112:600-7.

13 Santibañez S, Siegel V, O'Sullivan M, et al. Health communications and community mobilization during an Ebola response: partnerships with community and faith-based organizations. Public Health Rep 2015;130:128-33.

14 Roess AA, Di Peppi R, Kinzoni EA, et al. Knowledge gained and retained from a Video-Centered, community-based intervention for Ebola prevention, Congo. J Health Commun 2017;22:913-22.

15 Merkley E, Loewen PJ. Anti-intellectualism and information preferences during the COVID-19 pandemic. Nat Hum Behav 2021;5:706-15.

16 Mitchell T, Massoudi M, Swerdlow DL, et al. Swine flu in college: early campus response to outbreak control measures. Am J Health Behav 2014;38:448-64.

17 Menon KU. SARS revisited: managing "outbreaks" with "communications". Ann Acad Med Singap 2006;35:361-7.

18 Vaughan E, Tinker T. Effective health risk communication about pandemic influenza for vulnerable populations. Am J Public Health 2009;99 Suppl 2:S324-32.

19 Lin L, Savoia E, Agboola F, et al. What have we learned about communication inequalities during the $\mathrm{H} 1 \mathrm{~N} 1$ pandemic: a systematic review of the literature. BMC Public Health 2014;14:484.

20 Freiman AJ, Montgomery JP, Green JJ, et al. Did H1N1 influenza prevention messages reach the vulnerable population along the Mississippi Gulf Coast? J Public Health Manag Pract 2011;17:52-8.

21 Qian X, Ren R, Wang Y, et al. Fighting against the common enemy of COVID-19: a practice of building a community with a shared future for mankind. Infect Dis Poverty 2020;9:34.

22 Teasdale E, Yardley L. Understanding responses to government health recommendations: public perceptions of government advice for managing the $\mathrm{H} 1 \mathrm{~N} 1$ (swine flu) influenza pandemic. Patient Educ Couns 2011;85:413-8.
23 Davis MDM, Stephenson N, Lohm D, et al. Beyond resistance: social factors in the general public response to pandemic influenza. BMC Public Health 2015;15:436.

24 Daellenbach K, Parkinson J, Krisjanous J. Just how prepared are you? An application of marketing segmentation and theory of planned behavior for disaster preparation. Journal of Nonprofit \& Public Sector Marketing 2018;30:413-43.

25 Qu W, Chu C, Hou X. A Comparison of China's risk communication in response to SARS and $\mathrm{H} 7 \mathrm{~N} 9$ using principles drawn from international practice. Disaster Med 2018;12:587-98.

26 Idoiaga N, De Montes LG, Valencia J-F. Communication and representation of risk in health crises: the influence of framing and group identity. Revista de Psicologia Social 2016;31:59-74.

27 Jones SC, Waters L, Holland O, et al. Developing pandemic communication strategies: preparation without panic. J Bus Res 2010;63:126-32.

28 Hickey JE, Gagnon AJ, Jitthai N. Knowledge about pandemic influenza preparedness among vulnerable migrants in Thailand. Health Promot Int 2016;31:124-32.

29 Bonwitt J, Dawson M, Kandeh M, et al. Unintended consequences of the 'bushmeat ban' in West Africa during the 2013-2016 Ebola virus disease epidemic. Soc Sci Med 2018;200:166-73.

30 Aburto NJ, Pevzner E, Lopez-Ridaura R, et al. Knowledge and adoption of community mitigation efforts in Mexico during the 2009 H1N1 pandemic. Am J Prev Med 2010;39:395-402.

31 Lapka C, Jupka K, Wray RJ, et al. Applying cognitive response testing in message development and pre-testing. Health Educ Res 2008;23:467-76.

32 Gray L, MacDonald C, Mackie B, et al. Community responses to communication campaigns for influenza $A(\mathrm{H} 1 \mathrm{~N} 1)$ : a focus group study. BMC Public Health 2012;12:205

33 Hickey J, Gagnon AJ, Jitthai N. Pandemic preparedness: perceptions of vulnerable migrants in Thailand towards WHO-recommended nonpharmaceutical interventions: a cross-sectional study. BMC Public Health 2014;14:665.

34 Seltzer EK, Jean NS, Kramer-Golinkoff E, et al. The content of social media's shared images about Ebola: a retrospective study. Public Health 2015;129:1273-7.

35 Toppenberg-Pejcic D, Noyes J, Allen T, et al. Emergency risk communication: lessons learned from a rapid review of recent gray literature on Ebola, Zika, and yellow fever. Health Commun 2019;34:437-55.

36 Tully M, Dalrymple KE, Young R. Contextualizing nonprofits' use of links on Twitter during the West African Ebola virus epidemic. Commun Stud 2019;70:313-31.

37 Winters M, Jalloh MF, Sengeh P, et al. Risk communication and Ebola-Specific knowledge and behavior during 2014-2015 outbreak, Sierra Leone. Emerg Infect Dis 2018;24:336-44.

38 Avery EJ, Kim S. Anticipating or precipitating crisis? health agencies may not be heeding best practice advice in avian flu press releases. Journal of Public Relations Research 2009;21:187-97.

39 Davis OL, Fante RM, Jacobi LL. The effectiveness of sign prompts to increase hand washing behaviors in restrooms. North American Journal of Psychology 2013;15:565-76.

40 van der Weerd W, Timmermans DR, Beaujean DJ, et al. Monitoring the level of government trust, risk perception and intention of the general public to adopt protective measures during the influenza A $(\mathrm{H} 1 \mathrm{~N} 1)$ pandemic in The Netherlands. BMC Public Health 2011;11:575.

41 Li R, Xie R, Yang C, et al. Perceptions on the risk communication strategy during the 2013 avian influenza A/H7N9 outbreak in humans in China: a focus group study. Western Pac Surveill Response $J$ 2016;7:21-8.

42 Lohiniva A-L, Sane J, Sibenberg K, et al. Understanding coronavirus disease (COVID-19) risk perceptions among the public to enhance risk communication efforts: a practical approach for outbreaks, Finland, February 2020. Euro Surveill 2020;25:04.

43 Rogers WA, Street JM, Braunack-Mayer AJ, et al. Pandemic influenza communication: views from a deliberative forum. Health Expect 2009;12:331-42.

44 J. Holmes B, Henrich N, Hancock S, et al. Communicating with the public during health crises: experts' experiences and opinions. J Risk Res 2009;12:793-807.

45 Cole J, Watkins C. International employees' concerns during serious disease outbreaks and the potential impact on business continuity: lessons identified from the 2014-15 West African Ebola outbreak. J Bus Contin Emer Plan 2016;9:149-62.

46 Johnson BB, Slovic P. Fearing or fearsome Ebola communication? keeping the public in the dark about possible post-21-day symptoms and infectiousness could backfire. Health Risk Soc 2015;17:458-71. 
47 Bradshaw EL, Ryan R, Noetel M. Information safety assurances affect intentions to use COVID-19 contact tracing applications regardless of autonomy-supportive or controlling message framing. Front Psychol 2020;11:3772.

48 Dai B, Fu D, Meng G, et al. The effects of governmental and individual predictors on COVID-19 protective behaviors in China: a path analysis model. Public Adm Rev 202010.1111/puar.13236. [Epub ahead of print: 1905 2020].

49 Jhummon-Mahadnac ND, Knott J, Marshall C. A cross-sectional study of pandemic influenza health literacy and the effect of a public health campaign. BMC Res Notes 2012;5:377.

50 Sumo J, George G, Weah V, et al. Risk communication during disease outbreak response in post-Ebola Liberia: experiences in Sinoe and grand Kru counties. Pan Afr Med J 2019;33:4.

51 Abu-Akel A, Spitz A, West R. Who is listening? Spokesperson effect on communicating social and physical distancing measures during the COVID-19 pandemic. Front Psychol 2020;11:564434.

52 Goldberg MH, Gustafson A, Maibach EW, et al. Mask-Wearing increased after a government recommendation: a natural experiment in the U.S. during the COVID-19 pandemic. Frontiers in Communication 2020;5:44.

53 Berry TR, Wharf-Higgins J, Naylor PJ. Sars wars: an examination of the quantity and construction of health information in the news media. Health Commun 2007;21:35-44.

54 Wirz CD, Schwakopf JM, Brossard D. Self-reported compliance and attitudes about social distancing during the COVID-19 outbreak. OSF Preprints 2020;10.

55 Yardley L, Miller S, Schlotz W, et al. Evaluation of a web-based intervention to promote hand hygiene: exploratory randomized controlled trial. J Med Internet Res 2011;13:e107.

56 Miczo N, Danhour E, Lester KE, et al. Memorable messages and the H1N1 flu virus. West J Commun 2013;77:625-44.

57 Prati G, Pietrantoni L, Zani B. Compliance with recommendations for pandemic influenza $\mathrm{H} 1 \mathrm{~N} 1$ 2009: the role of trust and personal beliefs. Health Educ Res 2011;26:761-9.

58 McVernon J, Mason K, Petrony S, et al. Recommendations for and compliance with social restrictions during implementation of school closures in the early phase of the influenza A (H1N1) 2009 outbreak in Melbourne, Australia. BMC Infect Dis 2011;11:257.

59 Kavanagh AM, Bentley RJ, Mason KE, et al. Sources, perceived usefulness and understanding of information disseminated to families who entered home quarantine during the $\mathrm{H} 1 \mathrm{~N} 1$ pandemic in Victoria, Australia: a cross-sectional study. BMC Infect Dis 2011;11:2.

60 Chang C. News coverage of health-related issues and its impacts on perceptions: Taiwan as an example. Health Commun 2012;27:111-23.

61 Hoda J. Identification of information types and sources by the public for promoting awareness of middle East respiratory syndrome coronavirus in Saudi Arabia. Health Educ Res 2016;31:12-23.

$62 \mathrm{Rim} \mathrm{H}, \mathrm{JH} \mathrm{H}$, Kiousis S. The evidence of compelling arguments in agenda building: relationships among public information subsidies, media coverage, and risk perceptions during a pandemic outbreak Journal of Communication Management 2014;18:101-16.

63 Rimi NA, Sultana R, Ishtiak-Ahmed K, et al. Understanding the failure of a behavior change intervention to reduce risk behaviors for avian influenza transmission among backyard poultry raisers in rural Bangladesh: a focused ethnography. BMC Public Health 2016;16:858

64 Basnyat I, Lee ST. Framing of influenza A ( 11 N1) pandemic in a Singaporean newspaper. Health Promot Int 2015;30:942-53.

65 Updegraff JA, Emanuel AS, Gallagher KM, et al. Framing flu prevention--an experimental field test of signs promoting hand hygiene during the 2009-2010 H1N1 pandemic. Health Psychol 2011;30:295-9.

66 Sell TK, Boddie C, McGinty EE, et al. Media messages and perception of risk for Ebola virus infection, United States. Emerg Infect Dis 2017;23:108-11.

67 Liao Q, Cowling B, Lam WT, et al. Situational awareness and health protective responses to pandemic influenza $A(\mathrm{H} 1 \mathrm{~N} 1)$ in Hong Kong: a cross-sectional study. PLoS One 2010;5:e13350.

68 Bekalu MA, Bigman CA, McCloud RF, et al. The relative persuasiveness of narrative versus non-narrative health messages in public health emergency communication: evidence from a field experiment. Prev Med 2018;111:284-90.

69 Heffner J, Vives M-L, FeldmanHall O. Emotional responses to prosocial messages increase willingness to self-isolate during the COVID-19 pandemic. Pers Individ Dif 2021;170:110420.

70 Jordan J, Yoeli E, Rand D. Don't get it or don't spread it? Comparing self-interested versus prosocially framed COVID-19 prevention messaging. PsyArXiv 2020;10

71 Zikmund-Fisher BJ, Scherer AM, Knaus M, et al. Discussion of average versus extreme case severity in pandemic risk communications. Emerg Infect Dis 2017;23:706-8.

72 Menon KU, Goh KT. Transparency and trust: risk communications and the Singapore experience in managing SARS. Journal of Communication Management 2005;9:375-83.

73 Ajzen I. The theory of planned behavior. Organ Behav Hum Decis Process 1991;50:179-211.

74 Becker $\mathrm{MH}$. The health belief model and personal health behavior. Health Educ Monogr 1974;2:324-473.

75 Rogers RW. A protection motivation theory of fear appeals and attitude Change1. J Psychol 1975;91:93-114.

76 Schwartzer R. Self-Efficacy in the adoption and maintenance of health behaviours: theoretical approaches and a new model. Selfefficacy: Thought control of action Washington, DC: Hemisphere 1992:217-43.

77 Bandura A. Social cognitive theory: an agentic perspective. Annu Rev Psychol 2001;52:1-26.

78 Bandura A. Social cognitive theory of mass communication. Media Psychol 2001;3:265-99.

79 Hernández-García I, Giménez-Júlvez T. Assessment of health information about COVID-19 prevention on the Internet: Infodemiological study. JMIR Public Health Surveill 2020;6:e18717.

80 Hooker C, Capon A, Leask J. Communicating about risk: strategies for situations where public concern is high but the risk is low. Public Health Res Pract 2017;27:15.

81 Lin L, Jung M, McCloud RF, et al. Media use and communication inequalities in a public health emergency: a case study of 20092010 pandemic influenza A virus subtype H1N1. Public Health Rep 2014;129 Suppl 4:49-60.

82 Person B, Sy F, Holton K, et al. National center for Inectious diseases SCOT. fear and stigma: the epidemic within the SARS outbreak. Emerg Infect Dis 2004;10:358-63. 